\title{
Open-Label Real-World Pharmacy Purchaser Study of Two Fixed-Dose Combination OTC Cold Treatments; Wick MediNait, Wick DayMed Capsules or a Combination of Both
}

\author{
Gillian Phillipson1*, David Ramsey², Sue Aspley³, Ingo Fietze ${ }^{4}$ \\ ${ }^{1}$ Procter \& Gamble International Operations, Geneva Business Center, Geneva, Switzerland \\ ${ }^{2}$ Procter \& Gamble Health Care, Mason, OH, USA \\ ${ }^{3}$ Procter \& Gamble Technical Centres Ltd., Surrey, UK \\ ${ }^{4}$ Center of Sleep Medicine, Charité-Universitätsmedizin Berlin, Berlin, Germany \\ Email: *phillipson.gl@pg.com
}

How to cite this paper: Phillipson, G., Ramsey, D., Aspley, S. and Fietze, I. (2018) Open-Label Real-World Pharmacy Purchaser Study of Two Fixed-Dose Combination OTC Cold Treatments; Wick MediNait, Wick DayMed Capsules or a Combination of Both. Open Journal of Respiratory Diseases, 8, 43-62.

https://doi.org/10.4236/ojrd.2018.83006

Received: July 30, 2018

Accepted: August 28, 2018

Published: August 31, 2018

Copyright $\odot 2018$ by authors and Scientific Research Publishing Inc. This work is licensed under the Creative Commons Attribution International License (CC BY 4.0).

http://creativecommons.org/licenses/by/4.0/ (c) (i) Open Access

\begin{abstract}
Acute upper respiratory tract infections, i.e. common colds cause multiple symptoms and affect adults on average 2 - 4 times a year and children 6 - 8 times per year. Thousands of non-prescription, over-the-counter products are used by sufferers, aimed at relieving the various both er some symptoms such as cough, nasal congestion, sore throat, etc. This study evaluated real-world effectiveness of Wick MediNait or Wick DayMed medicines alone and together in a combination as a day and night treatment regimen. Adult cold sufferers were recruited in pharmacy by pharmacy staff following self-purchase of either Wick MediNait, Wick DayMed capsules, or the combination of DayMed and MediNait. Participants completed online questionnaires before and after product use (evening of the first day for DayMed and the morning after using MediNait at bedtime). For the primary endpoint "Wick MediNait was effective at relieving my symptoms", there was a statistically significant difference $(\mathrm{p}<0.0001)$ in the users who Strongly Agreed or Agreed vs. Neither Agreed or Disagreed, Disagreed or Strongly Disagreed. Further, all symptoms evaluated (cough, runny nose, nasal congestion, fever, headache, sore throat, muscle aches and pains, sneezing) were statistically improved after MediNait and DayMed alone and when both were used, and users were satisfied with both treatments. All treatments were well tolerated with minimal adverse event reporting. The diurnal use of DayMed capsules combined with the nocturnal use of MediNait allows cold sufferers to efficiently self-manage their symptom relief throughout the day and night.
\end{abstract}




\section{Keywords}

Wick MediNait, Real-World Use, User Satisfaction, Observational, Cold Symptom Relief, Sleep Quality

\section{Introduction}

Acute upper respiratory tract infection (URTI) is one of the most common infectious diseases worldwide affecting adults on average 2 - 4 times a year and children 6 - 8 times per year [1]. A large number of people make use of nonprescription, over-the-counter (OTC) medicines for themselves or their children [2], and many health professionals in primary care settings recommend them to their patients as a first-line treatment [3]. Self-care and self-medication have attracted considerable international healthcare policy interest, because they effectively reduce the burden on health services and have a significant positive impact on health systems and society at large [4].

Studies have shown that pharmacists play an important role in patient care and in the recommendation of non-prescription remedies [5]. Pharmacists are becoming more patient oriented and are bringing many positive changes to the lives of patients. There is considerable evidence that patient counselling and product recommendation enhances patient compliance, satisfaction and the establishment of a robust safety monitoring system.

Wick MediNait and DayMed are non-prescription medicines that relieve multiple symptoms of colds and influenza (the "flu"). Wick MediNait syrup is available in both Germany and Austria and is intended for use in the evening to relieve cold/flu symptoms to permit a good night's sleep. The active pharmaceutical ingredients (APIs) are paracetamol $(600 \mathrm{mg} / 30 \mathrm{~mL})$, dextromethorphan hydrobromide $(15 \mathrm{mg} / 30 \mathrm{~mL})$, ephedrine sulphate $(8 \mathrm{mg} / 30 \mathrm{~mL})$ and doxylamine succinate $(7.5 \mathrm{mg} / 30 \mathrm{~mL})$. Paracetamol is a non-aspirin pain reliever which can temporarily relieve common cold and flu symptoms of headache, minor aches and pains, fever and sore throat, and at typical OTC doses, is known to be a safe and effective analgesic [6]. Dextromethorphan is a cough suppressant that crosses the blood-brain-barrier and activates sigma opioid receptors on the cough centre in the central nervous system, thereby reducing the urge to cough due to minor throat and bronchial irritation [7] [8]. The addition of ephedrine sulphate to the combination achieves the benefit of nasal decongestion by exerting a vasoconstrictive effect on blood vessels [9]. Doxylamine succinate acts primarily as an antagonist of the histamine $\mathrm{H}_{1}$ receptor which is responsible for its antihistamine [10] and sedative effects [11] [12] although its concentration in MediNait is considerably lower than in sleep aid products (e.g., Hoggar ${ }^{\circledast}$ Night $=25 \mathrm{mg} /$ tablet). Further, doxylamine's anticholinergic effects in colds also reduce runny nose and sneezing [13] [14]. Outside of Germany and Austria Wick products are sold under the trade name Vicks. 
The efficacy of Wick MediNait on cold symptoms, and sleep quality was evaluated in a randomised, double-blind, placebo-controlled clinical study [15] [16]. The results support MediNait's role as an effective and convenient therapy for symptoms of nasal congestion, runny nose, cough and pain/body aches associated with the common cold and for increasing sleep quality disturbed by common cold symptoms.

Wick DayMed is a non-prescription medicine available in Germany to relieve multiple symptoms of colds/flu and is suitable for dosing during the day. The APIs are paracetamol (325 mg/capsule), dextromethorphan hydrobromide (10 $\mathrm{mg} /$ capsule) and phenylpropanolamine ( $12.5 \mathrm{mg} /$ capsule). A patient can take up to 2 capsules at one time and up to 8 capsules in a 24 hour period, with 4 - 6 hours between doses. DayMed capsules provide relief from pain and fever associated with colds/flu infection, and relief from irritating cough thanks to paracetamol and dextromethorphan hydrobromide respectively. Additionally, phenylpropanolamine binds to and activates alpha- and beta-adrenergic receptors in the mucosa of the respiratory tract resulting in vasoconstriction and reduction in swelling of nasal mucous membranes and reduction in tissue hyperemia, oedema, and nasal congestion [17] [18].

The APIs in both products have been well-established in the OTC colds medicine category with combination products appealing to colds sufferers who want products to offer relief from multiple symptoms. This study was conducted to evaluate real-world effectiveness of these medicines alone and together in a combination with each other as a day and night treatment regimen.

\section{Methods}

This national (Germany), prospective, non-comparative, open label, multi-site observational study was conducted in a manner consistent with the Declaration of Helsinki and EU Directives 2001/83/EC \& 2010/84/EU. Freiburger EthikKommission International provided Ethics approval. Pharmacist investigators previously known to the Clinical Research Organisation, Sourcia, were invited to participate as study sites by telephone. Adult cold sufferers were recruited directly in study pharmacy sites by pharmacy staff following self-purchase of either Wick MediNait, Wick DayMed capsules, or the combination of DayMed and MediNait from December 2016 to June 2017.

Participants were eligible to be enrolled in this study if they were aged $\geq 18$ years old, purchased Wick MediNait and/or Wick DayMed capsules for their own use, agreed to participate in the study after freely purchasing the Wick product(s), could access the internet at home and had signed the Informed Consent. Purchasers were not enrolled if they refused to participate in the study and were excluded if they had chronic cough or recurring respiratory symptoms and signs such as persistent cough or chronic respiratory symptoms/conditions, or had contra-indications or known hypersensitivity or allergy to any product ingredients as detailed in the Summary of Product Characteristics of the prod- 
uct(s) they purchased.

Participants completed online questionnaires before and after product use over one 24-hour period (evening of the first day for DayMed and the morning after using MediNait at bedtime). The primary endpoint was the assessment of MediNait user satisfaction via the question "MediNait was effective at relieving my symptoms". The questionnaires included individual cold symptom assessments using a 7-point Likert scale (none, very mild, mild, mild to moderate, moderate, moderate to severe, severe) [19] [20] [21]. Participants were asked to report any adverse event (AE) to their pharmacy. The pharmacist was responsible for completing an $\mathrm{AE}$ report form for each event.

The intent-to-treat (ITT) population was all participants who took at least one dose of the study product and was used for all efficacy analyses and other summarisations (AEs and demographics). The MediNait arm was summarised by itself. The specific DayMed questions from the combination arm were added to summarisations of the DayMed alone arm. Because DayMed was taken first in the combination of DayMed plus MediNait, DayMed data from the combination group were not affected by the other treatment thus were be pooled with DayMed alone to make a more comprehensive dataset to create a "Total DayMed user" population for analysis.

No formal sample size calculation was done for the study. A previous open-label study (different geography, different product, and different questionnaire) was assessed for sizing purposes [22]. The previous study indicated a small effect in cough $(\mathrm{N}>200$ needed for sample size) relative to the other assessments (typically $\mathrm{N}<100$ needed for sample size). The participant responses were analysed by binomial test categorising the question into 2 groups dependent on the question (e.g. Strongly Agree-Agree vs. Neutral-Strongly Disagree) and testing if differences existed between groups. Symptom data violated normality as anticipated so Wilcoxon signed-rank test was used for analysis. All statistical analyses were performed at a 2-sided, 0.05 level of significance and no multiple comparison adjustment was applied. Statistical analyses were conducted using "SAS" software version 9.4.

\section{Results}

\subsection{Baseline Data}

The study consisted of 219 MediNait alone users, 176 Total DayMed users, and 62 combination users, recruited from 57 pharmacies in Germany. The MediNait alone users had a mean age of 38.5 years, were $51 \%$ female, and had 2 days median length of symptoms at baseline (Table 1). Total DayMed users had a mean age of 38.9 years, were $46.6 \%$ female and had 2 days median length of symptoms at baseline (Table 1). Combination users had a mean age of 38.1 years, were $43.5 \%$ female and had 2 days median length of symptoms at baseline (Table 1).

Of the MediNait users at baseline, $91 \%$ experienced cough, $92 \%$ runny nose, 
93\% nasal congestion, $67 \%$ fever, $95 \%$ headache, $93 \%$ sore throat, $87 \%$ muscle aches and pains and 90\% sneezing. At baseline, 94\% Total DayMed users experienced cough, $88 \%$ runny nose, $93 \%$ nasal congestion, $71 \%$ fever, $96 \%$ headache, $94 \%$ sore throat, $94 \%$ muscle aches and pains and $85 \%$ sneezing. Of the combination users at baseline, $89 \%$ experienced cough, $82 \%$ runny nose, $94 \%$ nasal congestion, $61 \%$ fever, $98 \%$ headache, $90 \%$ sore throat, $89 \%$ muscle aches and pains and $81 \%$ sneezing.

Table 1. Summary of baseline characteristics.

\begin{tabular}{|c|c|}
\hline Parameter & \\
\hline \multicolumn{2}{|l|}{ Statistic/Category } \\
\hline MEDINAIT ALONE Users & $\mathrm{N}=219$ \\
\hline \multicolumn{2}{|l|}{ Age } \\
\hline Mean (SD) & $38.5(12.58)$ \\
\hline Median & 35 \\
\hline \multicolumn{2}{|l|}{ Gender } \\
\hline Female & $111(50.7 \%)$ \\
\hline Male & $108(49.3 \%)$ \\
\hline \multicolumn{2}{|l|}{ Length of Symptoms (Days) } \\
\hline Mean (SD) & $2.8(2.02)$ \\
\hline Median & 2 \\
\hline \multicolumn{2}{|c|}{ Pharmacist Recommend Wick MediNait } \\
\hline No & $106(48.4 \%)$ \\
\hline Yes & $113(51.6 \%)$ \\
\hline \multicolumn{2}{|l|}{ Rate Pharmacist Advice ${ }^{1}$} \\
\hline Not Applicable & $59(26.9 \%)$ \\
\hline Good & $75(34.2 \%)$ \\
\hline Very Good & $85(38.8 \%)$ \\
\hline TOTAL DAYMED Users & $\mathrm{N}=176$ \\
\hline \multicolumn{2}{|l|}{ Age } \\
\hline Mean (SD) & $38.9(11.41)$ \\
\hline Median & 35 \\
\hline \multicolumn{2}{|l|}{ Gender } \\
\hline Female & $82(46.6 \%)$ \\
\hline Male & $94(53.4 \%)$ \\
\hline \multicolumn{2}{|l|}{ Length of Symptoms (Days) } \\
\hline Mean (SD) & $2.6(1.65)$ \\
\hline Median & 2 \\
\hline \multicolumn{2}{|c|}{ Pharmacist Recommend Wick DayMed } \\
\hline No & $38(21.6 \%)$ \\
\hline Yes & $138(78.4 \%)$ \\
\hline \multicolumn{2}{|l|}{ Rate Pharmacist Advice ${ }^{1}$} \\
\hline Not Applicable & $20(11.4 \%)$ \\
\hline Fair & $2(1.1 \%)$ \\
\hline Good & $66(37.5 \%)$ \\
\hline Very Good & $88(50.0 \%)$ \\
\hline Parameter & \\
\hline
\end{tabular}




\begin{tabular}{|c|c|}
\hline Statistic/Category & \\
\hline DAYMED + MEDINAIT Users & $\mathrm{N}=62$ \\
\hline \multicolumn{2}{|l|}{ Age } \\
\hline Mean (SD) & $38.1(10.75)$ \\
\hline Median & 35 \\
\hline \multicolumn{2}{|l|}{ Gender } \\
\hline Female & $27(43.5 \%)$ \\
\hline Male & $35(56.5 \%)$ \\
\hline \multicolumn{2}{|l|}{ Length of Symptoms (Days) } \\
\hline Mean (SD) & $2.6(1.85)$ \\
\hline Median & 2 \\
\hline \multicolumn{2}{|l|}{ Pharmacist Recommend Wick DayMed } \\
\hline No & $19(30.6 \%)$ \\
\hline Yes & $43(69.4 \%)$ \\
\hline \multicolumn{2}{|l|}{ Rate Pharmacist Advice ${ }^{1}$} \\
\hline Not Applicable & $7(11.3 \%)$ \\
\hline Fair & $2(3.2 \%)$ \\
\hline Good & $25(40.3 \%)$ \\
\hline Very Good & $28(45.2 \%)$ \\
\hline
\end{tabular}

$\mathrm{N}=$ number of subjects within specified study. $\mathrm{n}(\%)=$ number and percentage of subjects within specified Parameter, study, and Category. ${ }^{1}$ Scale: not applicable, very bad, bad, fair, good, and very good. Categories with no responses are not included in the table.

About half of the participants purchased MediNait (52\%) following pharmacist recommendation (Table 1). Seventy-three percent of participants rated the pharmacists' advice as Good or Very Good. Amongst the Total DayMed users, $78 \%$ purchased the product because it was recommended by the pharmacist and $87 \%$ rated the pharmacists' advice as Good or Very Good. Approximately $69 \%$ of MediNait + DayMed purchased the 2 products upon pharmacist recommendation and $85 \%$ rated the pharmacists' advice as Good or Very Good.

\subsection{MediNait Alone}

After treatment with MediNait, there were highly significant changes in symptoms from before treatment $(\mathrm{p}<0.0001)$ with medians improving at least 2 units on the 7-point scale after a single dose of MediNait (exception cough median 1 unit of improvement but same significance) (Figure 1).

MediNait user satisfaction, including the primary endpoint, is presented in Table 2. The primary endpoint was met with $90 \%$ of the participants agreeing (Strongly Agreed or Agreed) that MediNait was effective at relieving their symptoms ( $\mathrm{p}<0.0001$ vs. Neither Agreed or Disagreed, Disagreed or Strongly Disagreed). There was a statistically significant difference $(\mathrm{p}<0.0001)$ in the number of participants who slept Much Better or Somewhat Better than those who slept As Well, Somewhat Worse, or Much Worse when they were asked to 
Table 2. Summary of user satisfaction with MediNait. Intent-to-treat Subjects.

\begin{tabular}{|c|c|}
\hline $\begin{array}{c}\text { Parameter } \\
\text { Category }\end{array}$ & $\begin{array}{c}\text { MediNait } \\
(\mathrm{N}=219) \mathrm{n}(\%)\end{array}$ \\
\hline Overall, with this cold last night, I did not sleep as well as usual & $\mathrm{p}<0.0001^{\mathrm{a}}$ \\
\hline Strongly Agree-Agree & $75(34.2 \%)$ \\
\hline Neither Agree or Disagree-Disagree-Strongly Disagree & $144(65.8 \%)$ \\
\hline Compared to the night before last, last night I slept & $\mathrm{p}<0.0001^{\mathrm{b}}$ \\
\hline Much Worse-Somewhat worse-About as well & $23(10.5 \%)$ \\
\hline Much Better-Somewhat Better & $196(89.5 \%)$ \\
\hline Wick MediNait was effective at relieving my symptoms & $\mathrm{p}<0.0001^{\mathrm{a}}$ \\
\hline Strongly Agree-Agree & $196(89.5 \%)$ \\
\hline Neither Agree or Disagree-Disagree-Strongly Disagree & $23(10.5 \%)$ \\
\hline Wick MediNait soothed my scratchy throat as soon as I took it & $\mathrm{p}=0.2485^{\mathrm{a}}$ \\
\hline Strongly Agree-Agree & $100(46.1 \%)$ \\
\hline Neither Agree or Disagree-Disagree-Strongly Disagree & $117(53.9 \%)$ \\
\hline Wick MediNait allowed me to have a good night sleep & $\mathrm{p}<0.0001^{\mathrm{a}}$ \\
\hline Strongly Agree-Agree & $194(89.4 \%)$ \\
\hline Neither Agree or Disagree-Disagree-Strongly Disagree & $23(10.6 \%)$ \\
\hline I felt better next morning having taken Wick MediNait & $\mathrm{p}<0.0001^{\mathrm{a}}$ \\
\hline Strongly Agree-Agree & $186(85.7 \%)$ \\
\hline Neither Agree or Disagree-Disagree-Strongly Disagree & $31(14.3 \%)$ \\
\hline I woke up refreshed having taken Wick MediNait & $\mathrm{p}<0.0001^{\mathrm{a}}$ \\
\hline Strongly Agree-Agree & $144(66.4 \%)$ \\
\hline Neither Agree or Disagree-Disagree-Strongly Disagree & $73(33.6 \%)$ \\
\hline I am satisfied with Wick MediNait & $\mathrm{p}<0.0001^{\mathrm{a}}$ \\
\hline Strongly Agree-Agree & $200(92.2 \%)$ \\
\hline Neither Agree or Disagree-Disagree-Strongly Disagree & $17(7.8 \%)$ \\
\hline I would buy Wick again when I have a cold & $\mathrm{p}<0.0001^{\mathrm{a}}$ \\
\hline Strongly Agree-Agree & $196(90.3 \%)$ \\
\hline Neither Agree or Disagree-Disagree-Strongly Disagree & $21(9.7 \%)$ \\
\hline I would recommend Wick MediNait to a friend & $\mathrm{p}<0.0001^{\mathrm{a}}$ \\
\hline Strongly Agree-Agree & $176(81.1 \%)$ \\
\hline Neither Agree or Disagree-Disagree-Strongly Disagree & $41(18.9 \%)$ \\
\hline I am happier with the effects of Wick MediNait than my usual cold medicine & $\mathrm{p}<0.0001^{\mathrm{a}}$ \\
\hline Strongly Agree-Agree & $146(67.3 \%)$ \\
\hline Neither Agree or Disagree-Disagree-Strongly Disagree & $71(32.7 \%)$ \\
\hline Wick MediNait relieves symptoms without being too strong & $\mathrm{p}<0.0001^{\mathrm{a}}$ \\
\hline Strongly Agree-Agree & $154(71.0 \%)$ \\
\hline Neither Agree or Disagree-Disagree-Strongly Disagree & $63(29.0 \%)$ \\
\hline
\end{tabular}




\section{Continued}

\begin{tabular}{|c|c|}
\hline $\begin{array}{l}\text { Parameter } \\
\text { Category }\end{array}$ & $\begin{array}{c}\text { MediNait } \\
(\mathrm{N}=219) \\
\mathrm{n}(\%)\end{array}$ \\
\hline Wick MediNait gave me fast cold relief & $\mathrm{p}<0.0001^{\mathrm{a}}$ \\
\hline Strongly Agree-Agree & $167(77.0 \%)$ \\
\hline Neither Agree or Disagree-Disagree-Strongly Disagree & $50(23.0 \%)$ \\
\hline $\begin{array}{l}\text { Wick MediNait was better at relieving pain than other } \\
\text { cold medicines I have used }\end{array}$ & $\mathrm{p}<0.0001^{\mathrm{a}}$ \\
\hline Strongly Agree-Agree & $129(68.6 \%)$ \\
\hline Neither Agree or Disagree-Disagree-Strongly Disagree & $59(31.4 \%)$ \\
\hline $\begin{array}{l}\text { Wick MediNait was better at relieving cough than other } \\
\text { cold medicines I have used }\end{array}$ & $\mathrm{p}=0.0646^{\mathrm{a}}$ \\
\hline Strongly Agree-Agree & $112(56.6 \%)$ \\
\hline Neither Agree or Disagree-Disagree-Strongly Disagree & $86(43.4 \%)$ \\
\hline $\begin{array}{l}\text { Wick MediNait was better at relieving runny nose than other } \\
\text { cold medicines I have used }\end{array}$ & $\mathrm{p}=0.7230^{\mathrm{a}}$ \\
\hline Strongly Agree-Agree & $102(51.3 \%)$ \\
\hline Neither Agree or Disagree-Disagree-Strongly Disagree & $97(48.7 \%)$ \\
\hline $\begin{array}{l}\text { Wick MediNait was better at relieving nasal congestion than other } \\
\text { cold medicines I have used }\end{array}$ & $\mathrm{p}=0.0288^{\mathrm{a}}$ \\
\hline Strongly Agree-Agree & $116(57.7 \%)$ \\
\hline Neither Agree or Disagree-Disagree-Strongly Disagree & $85(42.3 \%)$ \\
\hline Wick MediNait was more effective than other cold medicines I have used & $\mathrm{p}<0.0001^{\mathrm{a}}$ \\
\hline Strongly Agree-Agree & $153(70.5 \%)$ \\
\hline Neither Agree or Disagree-Disagree-Strongly Disagree & $64(29.5 \%)$ \\
\hline $\begin{array}{l}\text { Wick MediNait was more effective at relieving my symptoms overall compared to } \\
\text { other cold medication I have used }\end{array}$ & $\mathrm{p}<0.0001^{\mathrm{a}}$ \\
\hline Strongly Agree-Agree & $167(77.0 \%)$ \\
\hline Neither Agree or Disagree-Disagree-Strongly Disagree & $50(23.0 \%)$ \\
\hline $\begin{array}{l}\text { How did your Wick MediNait compare to other cold/flu products } \\
\text { you have used in the past }\end{array}$ & $\mathrm{p}<0.0001^{\mathrm{c}}$ \\
\hline Best cold/flu medicine-Better than most cold/flu medicines & $161(76.7 \%)$ \\
\hline $\begin{array}{l}\text { About the same as other cold/flu medicines-Worse than other } \\
\text { Cold/flu medicines }\end{array}$ & $49(23.3 \%)$ \\
\hline
\end{tabular}

$\mathrm{N}=$ number of subjects within specified study. $\mathrm{n}(\%)=$ number and percentage of subjects within specified Parameter, study, and Category. For questions regarding relieving pain, cough, runny nose, and nasal congestion, analysis was done in subjects with symptom present at baseline. a. Testing strongly agree-agree vs. neither agree or disagree, disagree and strongly disagree. b. Testing Much better-somewhat better vs. about as well, somewhat worse, and much worse. c. Testing best and better vs. about the same, worse, and worst cold/flu medicine. 


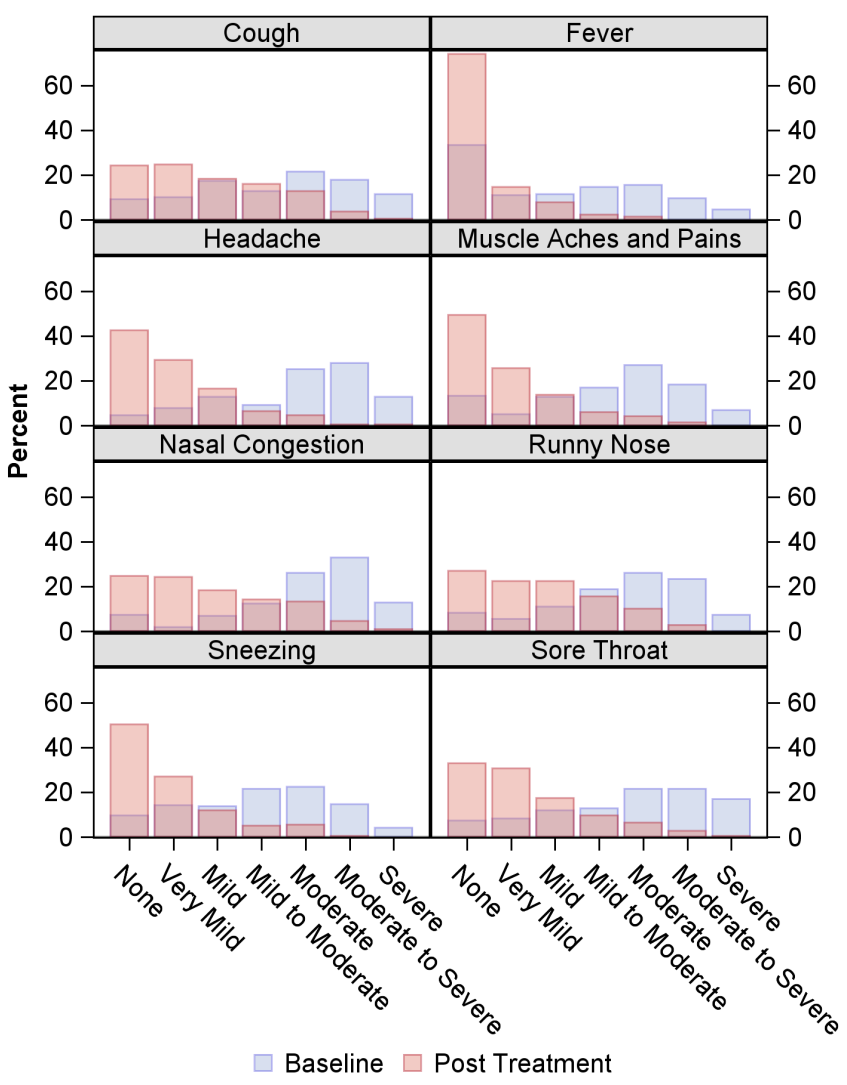

Figure 1. Summary of severity of symptoms before and after treatment with MediNait alone. There were highly statistically significant changes in symptoms from before treatment ( $\mathrm{p}<0.0001$ using Wilcoxon signed-rank test).

compare their night following treatment to the night before treatment. There was also a statistically significant difference $(\mathrm{p}<0.0001)$ in the number of participants who agreed (Strongly Agreed or Agreed) MediNait allowed a good night sleep compared to those that were neutral or who disagreed ( $p<0.0001$ vs. Neither Agreed or Disagreed, Disagreed or Strongly Disagreed).

There were also significantly more participants who agreed (Strongly Agreed or Agreed) they felt better the next morning having taken MediNait and who agreed they woke up refreshed having taken MediNait vs. those that did not ( $\mathrm{p}<$ 0.0001 vs. Neither Agreed or Disagreed, Disagreed or Strongly Disagreed).

For almost all questions, significantly more participants agreed with the positive user evaluation statements than those who provided a neutral-disagree response. The exceptions were "MediNait soothed my scratchy throat as soon as I took it" (46\% vs. $54 \%$ ), "MediNait was better at relieving cough than other cold medicines I have used" ( $57 \%$ vs. $43 \%$ ) and "MediNait was better at relieving runny nose than other cold medicines I have used" (51\% vs. $49 \%$ ). Finally, $76.7 \%$ of participants stated that MediNait was the Best or Better than most cold/flu medicines when they were asked to compare MediNait to other cold/flu products they had previously used ( $\mathrm{p}<0.0001$ vs. about the same as other cold/flu medicines-Worse than other Cold/flu medicines). 


\subsection{Total DayMed Users}

The average number of DayMed doses taken was 2.7 with a minimum of 1 to a maximum of 4 doses over the 24-hour study period (Table 3). After treatment with DayMed, there were highly significant improvements in the severity of symptoms compared to before treatment $(\mathrm{p}<0.0001)$, with medians improving at least 2 units on the 7-point scale (Figure 2).

User satisfaction with DayMed is presented in Table 4. All but one of the direct satisfaction evaluation statements were statistically significant in favour of the agreement responses (Strongly Agreed or Agreed) vs. those who provided a neutral-disagree response (Neither Agreed or Disagreed, Disagreed or Strongly Disagreed, $\mathrm{p}<0.0001$ ). The one statement which lacked significance was "I felt energised throughout the day with Wick DayMed" where $53.8 \%$ of the participants stated they agreed vs. $46.2 \%$ that stated they were neither in agreement or disagreed. Finally, $76.5 \%$ of participants stated that DayMed was the Best or Better than most cold/flu medicines when they were asked to compare DayMed to other daytime cold/flu products they had previously used ( $p<0.0001$ vs. about the same as other cold/flu medicines-Worse than other Cold/flu medicines).

\subsection{DayMed + MediNait Users}

The average number of DayMed doses taken was 2.4 with a minimum of 1 to a maximum of 4 doses over the 24 -hour study period (Table 3 ). There were highly significant changes in the severity of symptoms from before treatment $(\mathrm{p}<$ 0.0001 ) with medians improving at least 2 units on the 7-point scale after DayMed (Figure 3(a)) and MediNait (Figure 3(b)).

User satisfaction with DayMed plus MediNait is presented in Table 5. For all statements answered, there was a statistically significant difference in the number of participants who Strongly Agreed or Agreed vs. Neither Agreed or Disagreed, Disagreed or Strongly Disagreed.

\subsection{Safety Results}

Two AEs were reported by two participants; one reported having stomach pain but no information was provided by the pharmacy on the treatment used, and one reported a moderately severe cough while on Wick MediNait. These events were the only two spontaneously reported by the participants and noted by the pharmacists on AE report forms.

\section{Discussion}

Cold/flu combination medicines that contain several active ingredients, such as Wick MediNait and Wick DayMed capsules, offer effective relief from multiple symptoms of common cold and flu. Such medicines are considered safe, cost-effective and convenient when used as directed [23] and help overcome direct (resources) and indirect (productivity) losses associated with URTI [24]. 
Table 3. Dosing information-number of DayMed doses taken.

\begin{tabular}{cc}
\hline Parameter & \\
\hline Statistic/Category & $\mathrm{N}=176$ \\
\hline TOTAL DAYMED Users & $2.7(1.24)$ \\
\hline Mean (SD) & 1 \\
25\%-tile & 3 \\
Median & 4 \\
$75 \%$-tile & $1.0-4.0$ \\
Min-Max & $\mathrm{N}=62$ \\
\hline DAYMED + MEDINAIT Users & $2.4(1.27)$ \\
\hline Mean (SD) & 1 \\
Median & 2 \\
$75 \%$-tile & 4 \\
Min-Max & $1.0-4.0$ \\
\hline
\end{tabular}

$\mathrm{N}=$ number of subjects within specified study.

Table 4. Summary of user satisfaction after treatment with DayMed. DayMed total users: Intent-to-treat Subjects.

\begin{tabular}{|c|c|}
\hline $\begin{array}{c}\text { Parameter } \\
\text { Category }\end{array}$ & $\begin{array}{c}\text { DayMed } \\
(\mathrm{N}=176) \mathrm{n}(\%)\end{array}$ \\
\hline Wick DayMed was effective at relieving my symptoms & $\mathrm{p}<0.0001^{\mathrm{a}}$ \\
\hline Strongly Agree-Agree & $162(93.6 \%)$ \\
\hline Neither Agree or Disagree-Disagree-Strongly Disagree & $11(6.4 \%)$ \\
\hline I am satisfied with Wick DayMed & $\mathrm{p}<0.0001^{\mathrm{a}}$ \\
\hline Strongly Agree-Agree & $163(94.2 \%)$ \\
\hline Neither Agree or Disagree-Disagree-Strongly Disagree & $10(5.8 \%)$ \\
\hline I would buy Wick DayMed again when I have a cold & $\mathrm{p}<0.0001^{\mathrm{a}}$ \\
\hline Strongly Agree-Agree & $152(87.9 \%)$ \\
\hline Neither Agree or Disagree-Disagree-Strongly Disagree & $21(12.1 \%)$ \\
\hline I would recommend Wick DayMed to a friend & $\mathrm{p}<0.0001^{\mathrm{a}}$ \\
\hline Strongly Agree-Agree & $135(78.0 \%)$ \\
\hline Neither Agree or Disagree-Disagree-Strongly Disagree & $38(22.0 \%)$ \\
\hline I felt energised throughout the day with Wick DayMed & $\mathrm{p}=0.3230^{\mathrm{a}}$ \\
\hline Strongly Agree-Agree & $93(53.8 \%)$ \\
\hline Neither Agree or Disagree-Disagree-Strongly Disagree & $80(46.2 \%)$ \\
\hline
\end{tabular}




\section{Continued}

\begin{tabular}{|c|c|}
\hline Wick DayMed did not make me drowsy & $\mathrm{p}<0.0001^{\mathrm{a}}$ \\
\hline Strongly Agree-Agree & $140(80.9 \%)$ \\
\hline Neither Agree or Disagree-Disagree-Strongly Disagree & $33(19.1 \%)$ \\
\hline $\begin{array}{c}\text { Wick DayMed was better at relieving pain than other Daytime Cold medicines I } \\
\text { have used }\end{array}$ & $\mathrm{p}<0.0001^{\mathrm{a}}$ \\
\hline Strongly Agree-Agree & $112(68.7 \%)$ \\
\hline Neither Agree or Disagree-Disagree-Strongly Disagree & $51(31.3 \%)$ \\
\hline $\begin{array}{l}\text { Wick DayMed better at relieving cough than other daytime cold medicines I } \\
\text { have used }\end{array}$ & $\mathrm{p}<0.0001^{\mathrm{a}}$ \\
\hline Strongly Agree-Agree & $111(68.1 \%)$ \\
\hline Neither Agree or Disagree-Disagree-Strongly Disagree & $52(31.9 \%)$ \\
\hline $\begin{array}{l}\text { Wick DayMed better at relieving runny nose than other Daytime cold medicines } \\
\text { I have used }\end{array}$ & $\mathrm{p}<0.0001^{\mathrm{a}}$ \\
\hline Strongly Agree-Agree & $104(68.9 \%)$ \\
\hline Neither Agree or Disagree-Disagree-Strongly Disagree & $47(31.1 \%)$ \\
\hline $\begin{array}{l}\text { Wick DayMed better at relieving nasal congestion than other Daytime cold } \\
\text { medicines I have used }\end{array}$ & $\mathrm{p}<0.0001^{\mathrm{a}}$ \\
\hline Strongly Agree-Agree & $110(68.3 \%)$ \\
\hline Neither Agree or Disagree-Disagree-Strongly Disagree & $51(31.7 \%)$ \\
\hline $\begin{array}{l}\text { Wick DayMed was more effective than other Daytime cold medicines I have } \\
\text { used }\end{array}$ & $\mathrm{p}<0.0001^{\mathrm{a}}$ \\
\hline Strongly Agree-Agree & $127(73.4 \%)$ \\
\hline Neither Agree or Disagree-Disagree-Strongly Disagree & $46(26.6 \%)$ \\
\hline $\begin{array}{c}\text { Wick DayMed was more effective at relieving my symptoms overall compared to } \\
\text { other Daytime cold medicines I have used }\end{array}$ & $\mathrm{p}<0.0001^{\mathrm{a}}$ \\
\hline Strongly Agree-Agree & $131(75.7 \%)$ \\
\hline Neither Agree or Disagree-Disagree-Strongly Disagree & $42(24.3 \%)$ \\
\hline $\begin{array}{l}\text { Wick DayMed relieves my cold symptoms faster than other medicines I have } \\
\text { used }\end{array}$ & $\mathrm{p}<0.0001^{\mathrm{a}}$ \\
\hline Strongly Agree-Agree & $122(70.5 \%)$ \\
\hline Neither Agree or Disagree-Disagree-Strongly Disagree & $51(29.5 \%)$ \\
\hline Wick DayMed relieved my cold symptoms across my whole day & $\mathrm{p}<0.0001^{\mathrm{a}}$ \\
\hline Strongly Agree-Agree & $138(79.8 \%)$ \\
\hline Neither Agree or Disagree-Disagree-Strongly Disagree & $35(20.2 \%)$ \\
\hline $\begin{array}{l}\text { Wick DayMed relieved my fatigue/tiredness better than other medicines I have } \\
\text { used }\end{array}$ & $\mathrm{p}<0.0001^{\mathrm{a}}$ \\
\hline Strongly Agree-Agree & $114(65.9 \%)$ \\
\hline Neither Agree or Disagree-Disagree-Strongly Disagree & $59(34.1 \%)$ \\
\hline
\end{tabular}




\section{Continued}

How did your Wick DayMed compare to other daytime cold/flu products you have used

Best cold/flu medicine-Better than most cold/flu medicines $\mathrm{p}<0.0001^{\mathrm{b}}$

$127(76.5 \%)$

$39(23.5 \%)$

About the same as other cold/flu medicines-Worse than other Cold/flu medicines

$\mathrm{N}=$ number of subjects within specified study. $\mathrm{n}(\%)=$ number and percentage of subjects within specified Parameter, study, and Category. For questions regarding relieving pain, cough, runny nose, and nasal con-
gestion, analysis was done in subjects with symptom present at baseline. a. Testing strongly agree-agree vs. neither agree or disagree, disagree and strongly disagree. b. Testing best and better vs. about the same, worse, and worst cold/flu medicine.

Table 5. Summary of user satisfaction after treatment with DayMed plus MediNait. DayMed plus MediNait Users: Intent-to-treat Subjects.

\begin{tabular}{|c|c|}
\hline $\begin{array}{c}\text { Parameter } \\
\text { Category }\end{array}$ & $\begin{array}{c}\text { DayMed plus } \\
\text { MediNait } \\
(\mathrm{N}=58) \mathrm{n}(\%)\end{array}$ \\
\hline $\begin{array}{l}\text { The combination of Wick DayMed and Wick MediNait provided effective } \\
\text { day and night cold relief }\end{array}$ & $\mathrm{p}<0.0001^{\mathrm{a}}$ \\
\hline Strongly Agree-Agree & $54(93.1 \%)$ \\
\hline Neither Agree or Disagree-Disagree-Strongly Disagree & $4(6.9 \%)$ \\
\hline $\begin{array}{l}\text { Wick DayMed and Wick MediNait are effective partners for round the clock } \\
\text { cold relief }\end{array}$ & $\mathrm{p}<0.0001^{\mathrm{a}}$ \\
\hline Strongly Agree-Agree & $53(91.4 \%)$ \\
\hline Neither Agree or Disagree-Disagree-Strongly Disagree & $5(8.6 \%)$ \\
\hline $\begin{array}{l}\text { The combination of Wick DayMed and Wick MediNait was more effective } \\
\text { than other cold therapy I have used }\end{array}$ & $\mathrm{p}<0.0001^{\mathrm{a}}$ \\
\hline Strongly Agree-Agree & $44(75.9 \%)$ \\
\hline Neither Agree or Disagree-Disagree-Strongly Disagree & $14(24.1 \%)$ \\
\hline $\begin{array}{l}\text { The combination of Wick DayMed and Wick MediNait was more effective at } \\
\text { relieving my symptoms overall compared to other cold therapy I have used }\end{array}$ & $\mathrm{p}=0.0002^{\mathrm{a}}$ \\
\hline Strongly Agree-Agree & $43(74.1 \%)$ \\
\hline Neither Agree or Disagree-Disagree-Strongly Disagree & $15(25.9 \%)$ \\
\hline I would recommend Wick MediNait/DayMed therapy to a friend & $\mathrm{p}<0.0001^{\mathrm{a}}$ \\
\hline Strongly Agree-Agree & $48(82.8 \%)$ \\
\hline Neither Agree or Disagree-Disagree-Strongly Disagree & $10(17.2 \%)$ \\
\hline I would buy Wick DayMed/MediNait again & $\mathrm{p}<0.0001^{\mathrm{a}}$ \\
\hline Strongly Agree-Agree & $57(98.3 \%)$ \\
\hline Neither Agree or Disagree-Disagree-Strongly Disagree & $1(1.7 \%)$ \\
\hline
\end{tabular}

$\mathrm{N}=$ number of subjects within specified study. $\mathrm{n}(\%)=$ number and percentage of subjects within specified Parameter, study, and Category. a. Testing strongly agree-agree vs. neither agree or disagree, disagree and strongly disagree. 


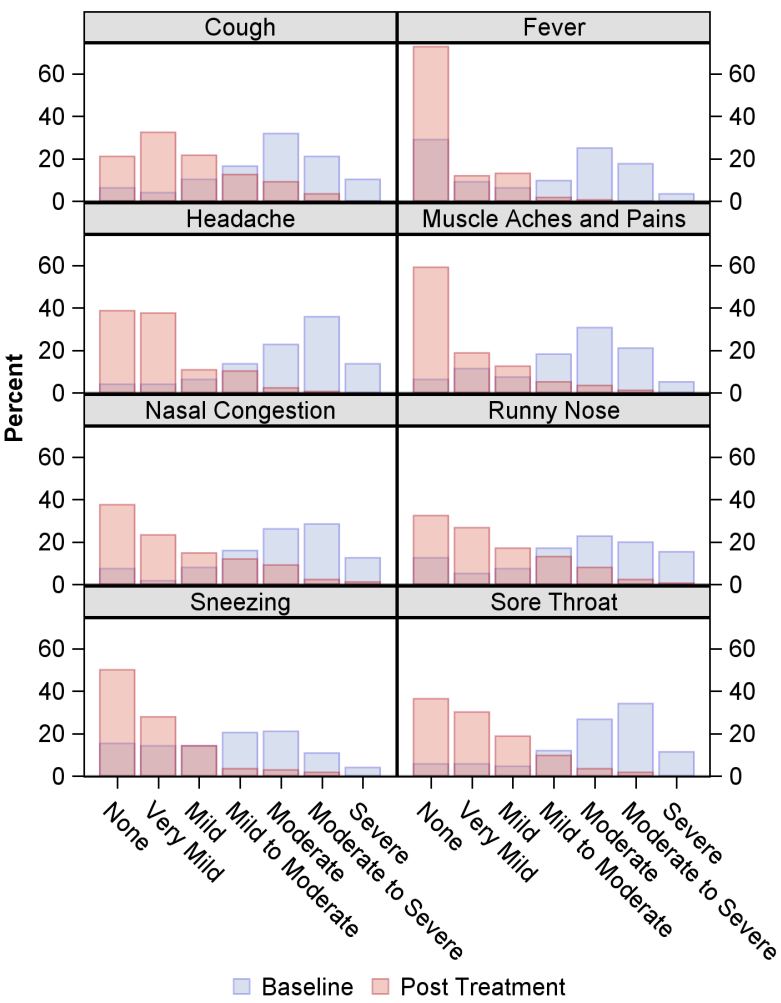

Figure 2. Summary of severity of symptoms before and after treatment with DayMed (Total Users). There were highly statistically significant changes in symptoms from before treatment ( $\mathrm{p}<0.0001$ using Wilcoxon signed-rank test).

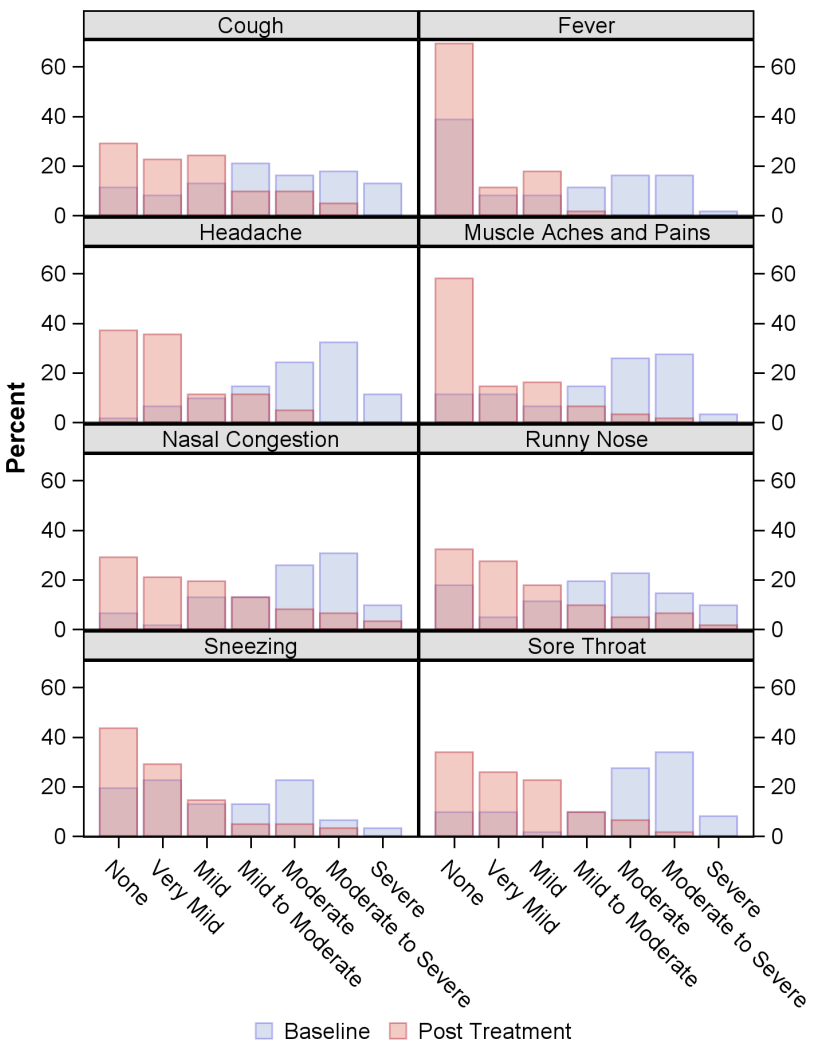

(a) 


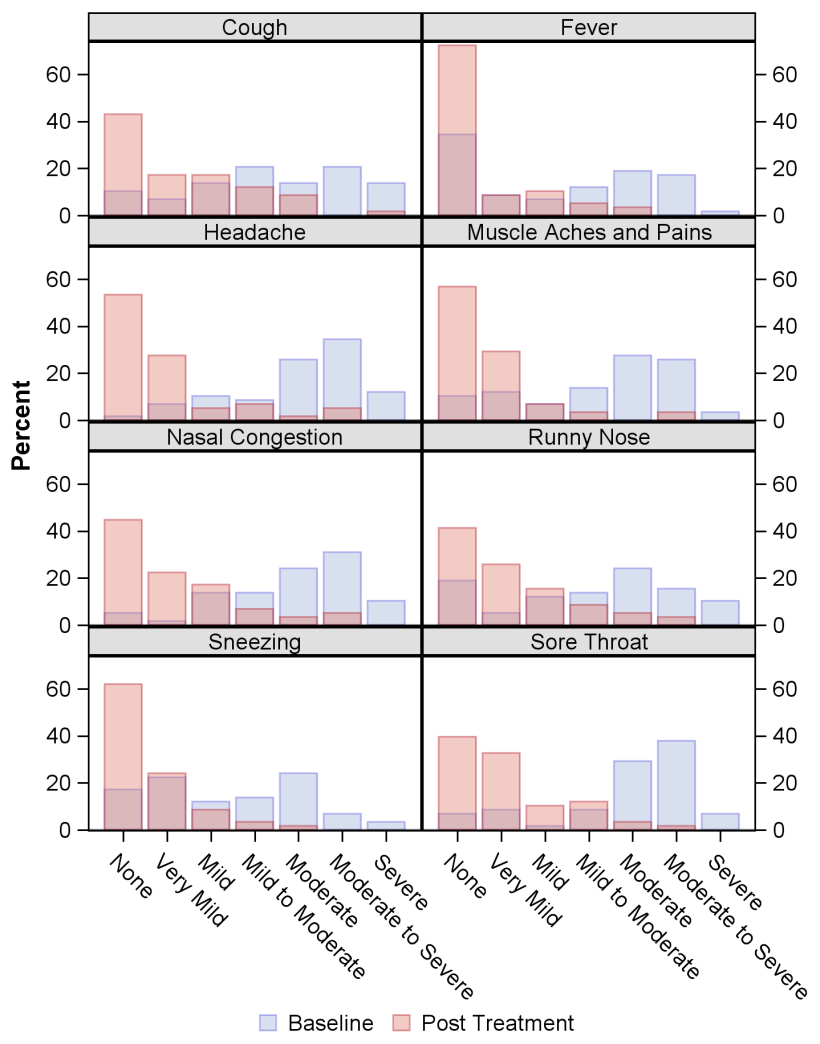

(b)

Figure 3. (a) Summary of Severity of Symptoms Before and After Treatment with DayMed (Combination Users); (b) Summary of Severity of Symptoms Before and After Treatment with MediNait (Combination Users). There were highly statistically significant changes in symptoms from before treatment $(\mathrm{p}<$ 0.0001 using Wilcoxon signed-rank test).

Observational studies offer an opportunity to gather evidence of a marketed drug working in real-world heterogeneous populations that can complement clinical evidence and post-marketing surveillance. While real-world data will never replace randomised controlled double-blind data for the registration of drugs, the value and acceptance of real world data in the assessment of in-use performance of medicines is an area of growing interest to regulators [25].

This observational study set out to evaluate the real-world experience of users purchasing Wick MediNait and DayMed capsules separately and in combination. The reduction in multiple symptom severity was significant for all treatment regimens and represented, for most symptoms, a median reduction of 2 units on the 7-point scale (MediNait "Cough" was the only exception with a smaller but significant severity reduction). The larger changes of 2 units likely represent clinically meaningful reductions as this would be approximately comparable to moving from a "Moderate" to "Mild" severity for example.

The user purchaser assessment for MediNait was completed upon waking the following morning following a single $30 \mathrm{~mL}$ dose at bedtime the evening before. The user purchaser assessment for DayMed was requested for the evening fol- 
lowing purchase and normal use hence it was possible to obtain information about the dosing frequency. Participants were compliant with the product dosing information with a maximum of 4 doses noted in the study evaluation period (24 hours).

Cold and flu infection, and symptoms such as nasal congestion and coughing, to negatively impact sleep [26] [27] and this can be particularly frustrating for sufferers. Sleep and immunity are believed to have a reciprocal relationship where changes in the immune system cause changes in sleep and, conversely, sleep has an important role in restoring the immune system [28]. Cold/flu sufferers often turn to OTC medication to relieve symptoms to help them get the respite they need to sleep [27]. Wick MediNait is a frequent choice for nighttime relief of multiple symptoms of cold/flu in Germany. Clinical data demonstrates MediNait is effective in relieving symptoms and can improve sleep quality [15] yet there is a still a concern that the product is too sedating due to the use of doxylamine succinate, a first-generation anti-histamine used in the product for the relief of runny nose and sneezing. In the present study, MediNait user purchasers reported relief of symptoms of nasal congestion, runny nose, cough and pain/body aches associated with the common cold and the majority agreed that the product helped them sleep better (89\%) and allowed them to have a good night's sleep (89\%). On top, many more users agreed they felt better the next morning (86\%), woke up refreshed (66\%), stated they believe MediNait relieves symptoms without being too strong (71\%) and no adverse events related to morning grogginess were noted. Together these findings suggest that MediNait helped users sleep well through effective relief of multiple cold/flu symptoms and not through a heavy sedative effect.

User satisfaction with DayMed and the combination were also very positive overall and the fact that the study could recruit the latter group demonstrates that sufferers are indeed purchasing the combination of both Wick DayMed capsules and MediNait for use together as a regimen approach to self-manage symptoms in Germany. The value of this approach was also confirmed by the user responses e.g. 93\% stating agreement to "The combination of Wick DayMed and Wick MediNait provided effective day and night cold relief".

There are several limitations of the study such as the sparse demographic information, unknown co-morbidities, unknown drug history along with the influence of self-selected product purchase. It is likely that the latter has the largest bearing as the participants were unlikely to select to purchase and use a treatment that they believed would be ineffective. This patient held belief in the effectiveness of their chosen treatment may indeed have influenced the overall scoring and without a placebo comparator it is unfeasible to measure the true drug effect with this type of study design. Viewed through a different lens this limitation could be considered a strength as the study more accurately reflects the end user assessment of treatment effectiveness and satisfaction in the real world, where placebo controls do not exist and consumers select and purchase treatments without the intervention of a healthcare professional. Despite a concern 
over the potential for inflated end user scoring, the magnitude of the treatment effect and satisfaction scoring confirms that users likely experienced a clinically relevant benefit of treatment. This data, combined with the observation of treatment compliance and tolerability, provides valuable information about MediNait and DayMed and emphasises the value of multi-active combination cold therapy to patients in heterogenous real-world community populations.

\section{Conclusion}

Wick MediNait and DayMed capsules, two representatives of a combination therapy for common cold, are effective treatments for multiple cold symptoms. This real world open label observational study demonstrates that both treatments alone and in combination were effective at relieving all colds symptoms evaluated (cough, runny nose, nasal congestion, fever, headache, sore throat, muscle aches and pains, sneezing) with improvement that was statistically and clinically significant. User satisfaction was also high for all treatment arms with many questions achieving high statistical significance and all treatments were well tolerated with minimal AE reporting. The diurnal use of DayMed capsules combined with the nocturnal use of MediNait allows cold sufferers to efficiently self-manage their symptom relief throughout the day and at night that helps supports natural recovery in part through enabling a good night's sleep.

\section{Acknowledgements}

The authors gratefully acknowledge the assistance of the Clinical Research Organisations Bioscience S. A., with its registered office at Kraszewskiego 1 St, 85 240 Bydgoszcz, Poland, and Sourcia, with its registered office at Zuidhaven 9, 4761 CR Zevenbergen, Netherlands in study execution. The authors also wish to thank Judith M. Pepin, PhD for medical writing services.

\section{Declarations}

This work was sponsored in full by Procter \& Gamble. At the time of conducting the study and preparing this manuscript, G. Phillipson, D. Ramsey and S. Aspley were full-time employees of The Procter \& Gamble Company and may have stock and/or stock options in the company. I. Fietze is an independent Professor of Sleep Medicine at Charité-Universitätsmedizin Berlin and is a paid Consultant to Procter \& Gamble. G. Phillipson and D. Ramsey were responsible for the interpretation of study results and G. Phillipson contributed to the drafting of the manuscript. All attributed authors participated in a review of the manuscript.

\section{Conflicts of Interest}

The authors declare no conflicts of interest regarding the publication of this paper. 


\section{References}

[1] Heikkinen, T. and Järvinen A. (2003) The Common Cold. The Lancet, 361, 51-59. https://doi.org/10.1016/S0140-6736(03)12162-9

[2] Eccles, R., Turner, R.B. and Dicpinigaitis, P.V. (2016) Treatment of Acute Cough Due to the Common Cold: Multi-Component, Multi-Symptom Therapy Is Preferable to Single-Component, Single-Symptom Therapy-A Pro/Con Debate. Lung, 194, 15-20. https://doi.org/10.1007/s00408-015-9808-5

[3] Eckel, N., Sarganas, G., Wolf, I.-K. and Knopf, H. (2014) Pharmacoepidemiology of Common Colds and Upper Respiratory Tract Infections in Children and Adolescents in Germany. BMC Pharmacology and Toxicology, 15, 44. https://doi.org/10.1186/2050-6511-15-44

[4] Proprietary Association of Great Britain (2018) A Long-Term Vision for Self Care: Interim White Paper.

https://www.pagb.co.uk/content/uploads/2018/07/A-long-term-vision-for-self-careinterim-white-paper.pdf

[5] Adams, M.L. and Blouin, R.A. (2017) The Role of the Pharmacist in Health Care Expanding and Evolving. North Carolina Medical Journal, 78, 165-167.

https://doi.org/10.18043/ncm.78.3.165

[6] Bunchorntavakul, C. and Reddy, K.R. (2013) Acetaminophen-Related Hepatotoxicity. Clinical Liver Disease, 17, 587-607. https://doi.org/10.1016/j.cld.2013.07.005

[7] Pavesi, L., Subburaj, S. and Porter-Shaw, K. (2001) Application and Validation of a Computerized Cough Acquisition System for Objective Monitoring of Acute Cough: A Meta-Analysis. Chest, 120, 1121-1128. https://doi.org/10.1378/chest.120.4.1121

[8] Taylor, C.P., Traynelis, S.F., Siffert, J., Pope, L.E. and Matsumoto, R.R. (2016) Pharmacology of Dextromethorphan: Relevance to Dextromethorphan/Quinidine (Nuedexta ${ }^{\oplus}$ ) Clinical Use. Pharmacology \& Therapeutics, 164, 170-182. https://doi.org/10.1016/j.pharmthera.2016.04.010

[9] McLaurin, J.W., Shipman, W.F. and Rosedale Jr., R. (1961) Oral Decongestants. A Double Blind Comparison Study of the Effectiveness of Four Sympathomimetic Drugs: Objective and Subjective. The Laryngoscope, 71, 54-67. https://doi.org/10.1288/00005537-196101000-00007

[10] Vande Griend, J.P. and Anderson, S.L. (2012) Histamine-1 Receptor Antagonism for Treatment of Insomnia. Journal of the American Pharmacists Association, 52, e210-e219. https://doi.org/10.1331/JAPhA.2012.12051

[11] Smith, G.M. and Smith, P.H. (1985) Effects of Doxylamine and Acetaminophen on Postoperative Sleep. Clinical Pharmacology \& Therapeutics, 37, 549-557. https://doi.org/10.1038/clpt.1985.87

[12] Melnikov, A.Y., Lavrik, S.Y., Bikbulatova, L.F., Raginene, I.G., Ivanova, Y.A. and Zakharov, A.V. (2017) Effectiveness of Reslip (Doxylamine) in Short-Term Insomnia: Multicenter Comparative Randomized Study. Zhurnal Nevrologii i Psihiatrii imeni S.S. Korsakova, 117, 56-59.

[13] Eccles, R., Van Cauwenberge, P., Tetzloff, W. and Borum, P. (1995) A Clinical Study to Evaluate the Efficacy of the Antihistamine Doxylamine Succinate in the Relief of Runny Nose and Sneezing Associated with Upper Respiratory Tract Infection. Journal of Pharmacy and Pharmacology, 47, 990-993. https://doi.org/10.1111/j.2042-7158.1995.tb03283.x

[14] D’Agostino Sr., R.B., Weintraub, M., Russell, H.K., Stepanians, M., D’Agostino Jr., 
R.B., Cantilena Jr., L.R., Graumlich, J.F., Maldonado, S., Honig, P. and Anello, C. (1998) The Effectiveness of Antihistamines in Reducing the Severity of Runny Nose and Sneezing: A Meta-Analysis. Clinical Pharmacology \& Therapeutics, 64, 579-596. https://doi.org/10.1016/S0009-9236(98)90049-2

[15] Mizoguchi, H., Wilson, A., Jerdack, G.R., Hull, J.D., Goodale, M., Grender, J.M. and Tyler, B.A. (2007) Efficacy of a Single Evening Dose of a Syrup Containing Paracetamol, Dextromethorphan Hydrobromide, Doxylamine Succinate and Ephedrine Sulfate in Subjects with Multiple Common Cold Symptoms. International Journal of Clinical Pharmacology and Therapeutics, 45, 230-236. https://doi.org/10.5414/CPP45230

[16] De Sutter, A.I., van Driel, M.L., Kumar, A.A., Lesslar, O. and Skrt, A. (2012) Oral Antihistamine-Decongestant-Analgesic Combinations for the Common Cold. Cochrane Library: Cochrane Reviews, 15, CD004976.

[17] Lea, P. (1984) A Double-Blind Controlled Evaluation of the Nasal Decongestant Effect of Day Nurse in the Common Cold. Journal of International Medical Research, 12, 124-127. https://doi.org/10.1177/030006058401200211

[18] Johnson, D.A. and Hricik, J.G. (1993) The Pharmacology of Alpha-Adrenergic Decongestants. Pharmacotherapy, 13, 110S-115S.

[19] Likert, R. (1932) A Technique for the Measurement of Attitudes. Archives of Psychology, 22, 5-55.

[20] Landis, J.R. and Koch, G.G. (1977) The Measurement of Observer Agreement for Categorical Data. Biometrics, 33, 159-174. https://doi.org/10.2307/2529310

[21] Norman, G. (2010) Likert Scales, Levels of Measurement and the "Laws" of Statistics. Advances in Health Sciences Education, 15, 625-632. https://doi.org/10.1007/s10459-010-9222-y

[22] Phillipson, G.L., Hull, J.D. and Samoliński, B. (2017) Observational Study of a Multi-Active Ingredient Over-the-Counter Cold Remedy Following Active Pharmacist Recommendation. Open Journal of Respiratory Diseases, 7, 41-52. https://doi.org/10.4236/ojrd.2017.71005

[23] Eccles, R., Fietze, I. and Rose, U.B. (2014) Rationale for Treatment of Common Cold and Flu with Multi-Ingredient Combination Products for Multi-Symptom Relief in Adults. Open Journal of Respiratory Diseases, 4, 73-82.

https://doi.org/10.4236/ojrd.2014.43011

[24] Bramley, T.J., Lerner, D. and Sames, M. (2002) Productivity Losses Related to the Common Cold. Journal of Occupational and Environmental Medicine, 44, 822-829. https://doi.org/10.1097/00043764-200209000-00004

[25] FDA (2017) Use of Real-World Evidence to Support Regulatory Decisions-Making for Medical Devices: Guidance for Industry and Food and Drug Administration Staff.

https://www.fda.gov/downloads/medicaldevices/deviceregulationandguidance/guid ancedocuments/ucm513027.pdf

[26] Drake, C.L., Roehrs, T.A., Royer, H., Koshorek, G., Turner, R.B. and Roth, T. (2000) Effects of an Experimentally Induced Rhinovirus Cold on Sleep, Performance, and Daytime Alertness. Physiology \& Behavior, 71, 75-81. https://doi.org/10.1016/S0031-9384(00)00322-X

[27] Hull, J.D., Barton, I.P., Torgersen, J. and McNeil, C.M. (2013) Survey of the Experience and Impact of Acute Upper Respiratory Tract Infections on People in Six Countries in the 2011/2012 Common Cold and Flu Season. Open Journal of Respi- 
ratory Diseases, 3, 175-187. https://doi.org/10.4236/ojrd.2013.34026

[28] Bryant, P.A., Trinder, J. and Curtis, N. (2004) Sick and Tired: Does Sleep Have a Vital Role in the Immune System? Nature Reviews Immunology, 4, 457-467. https://doi.org/10.1038/nri1369 